\title{
Tooth brushing inhibits oral bacteria in dogs
}

\author{
Kazuhiro WATANABE ${ }^{1) *}$, Kotaro HAYASHI ${ }^{1)}$, Saku KIJIMA ${ }^{1)}$, Chie NONAKA $^{1)}$ and Kazuaki YAMAZOE ${ }^{1)}$ \\ 1) Laboratory of Veterinary Surgery, Clinical Veterinary Medicine, Joint Department of Veterinary Medicine, Faculty of Applied Biological \\ Sciences, Gifu University, 1-1 Yanagido, Gifu 501-1193, Japan
}

(Received 11 April 2014/Accepted 8 May 2015/Published online in J-STAGE 21 May 2015)

ABSTRACT. In this study, scaling, polishing and daily tooth brushing were performed in 20 beagle dogs, and the number of oral bacteria was determined using a bacterial counter. The dogs were randomized into the scaling (S), scaling + polishing (SP), scaling + tooth daily brushing (SB) and scaling + polishing + tooth daily brushing (SPB) groups. Samples were collected from the buccal surface of the maxillary fourth premolars of the dogs immediately after scaling and every week thereafter from weeks 1 to 8 . Throughout the study, the number of bacteria was significantly lower in the SB and SPB groups compared with the S group. The findings suggest that daily tooth brushing inhibited oral bacterial growth in the dogs.

KEY WORDS: canine, oral bacteria, polishing, scaling, tooth brushing

doi: 10.1292/jvms.14-0193; J. Vet. Med. Sci. 77(10): 1323-1325, 2015

The incidence of periodontal diseases increases in dogs when they become older, and approximately $80 \%$ of dogs have some stage of periodontal disease [5]. The most common procedure to treat periodontal diseases in dogs is scaling, which should be performed under general anesthesia. Owing to the risks of anesthesia, it may be difficult to obtain consent from the owners. Even if consent is obtained, the only treatment option for dogs with severe periodontal diseases is tooth extraction. Therefore, prevention is more important than treatment in case of periodontal diseases. The most common preventive measures are to eliminate the growing bacteria in the dental plaque and/or calculus and to inhibit bacterial colonization [7].

Effective prevention of periodontal diseases includes professional scaling and polishing by a veterinarian and tooth brushing by an owner [7]. Mechanical scaling removes dental calculi from tooth surfaces using tools, such as an ultrasonic scaler [2]. Polishing the tooth surfaces after scaling prevents colonization of bacteria [2]. Brushing with a tooth brush removes plaque on tooth surfaces, which may be the most effective way to reduce the number of bacteria [2]. It is reported that tooth brushing reduces oral bacteria up to approximately $70 \%$ in humans $[9,12]$. These findings suggest that polishing and brushing theoretically may reduce the number of oral bacteria in dogs as well as humans; however, it is not known whether professional scaling, polishing and daily brushing have a long-term effect on reduction of oral bacteria in dogs.

In this study, dogs received scaling, scaling and polishing,

*Correspondence to: Watanabe, K., Laboratory of Veterinary Surgery, Clinical Veterinary Medicine, Joint Department of Veterinary Medicine, Faculty of Applied Biological Sciences, Gifu University, 1-1 Yanagido, Gifu 501-1193, Japan.

e-mail: nabechan@gifu-u.ac.jp

C2015 The Japanese Society of Veterinary Science

This is an open-access article distributed under the terms of the Creative Commons Attribution Non-Commercial No Derivatives (by-nc-nd) License $<$ http://creativecommons.org/licenses/by-nc-nd/3.0/>. scaling and daily brushing, or scaling and polishing and daily brushing. Changes over time in the number of oral bacteria were measured to evaluate the effect of these procedures.

This study used 20 beagle dogs ( 7 males and 13 females, aged 1 to 6 years old and weighing $12.9 \pm 2.1 \mathrm{~kg}$ ) that were clinically healthy without severe periodontitis. The animals were equally randomized into 4 groups (5 dogs in each group), including the scaling group (S), polishing immediately after scaling group (SP), scaling followed by daily brushing group (SB) and polishing immediately after scaling and followed by daily brushing group (SPB). All the dogs were housed in the same location during the study period, fed the same dry food (TC-2, Oriental Yeast Co., Ltd., Tokyo, Japan) once a day and freely supplied with water. This study was approved by the Animal Care and Use Committee, Faculty of Applied Biological Sciences, Gifu University.

At the start of the experiment, a combination of medetomidine $(30 \mu \mathrm{g} / \mathrm{kg})$ and midazolam $(0.15 \mathrm{mg} / \mathrm{kg})$ was intravenously administered to all dogs for sedation. Supragingival calculi were removed using an ultrasonic scaler; removal of subgingival calculi and root planing were performed using a curette scaler. In addition, polishing was performed on the dogs in the SP and SPB groups using a tooth polishing paste, a polishing brush and a rubber cup. After completion of these procedures, atipamezole $(0.15 \mathrm{mg} / \mathrm{kg})$ was intravenously administered to reverse the sedative effect of medetomidine.

Teeth of the dogs in the SB and SPB groups were brushed using a tooth brush for dogs without anesthesia within 2 hr after eating every day. The rolling method was applied using a tap-water-soaked brush until the brush was clear of food debris. Each dog had their own tooth brush that was exchanged every 2 weeks. The brushing procedures were performed by the same operator.

Samples for determination of oral bacteria were collected from the teeth immediately after scaling (Week 0 ) and every week from Weeks 1 to 8 in order to determine the number of oral bacteria. After a 6-hr fasting (both food and water) period, a combination of medetomidine $(30 \mu \mathrm{g} / \mathrm{kg})$ and midazolam $(0.15 \mathrm{mg} / \mathrm{kg})$ was intramuscularly administered to the dogs 
for sedation, and samples were collected. A weight of $20 \pm 5$ $\mathrm{g}$ was loaded on the buccal surface of the left and right maxillary fourth premolars by using a sample collection device under constant pressure with a sterile cotton swab. The buccal surface was rubbed back and forth 3 times using the cotton swab in a parallel position to the surface to collect samples (Fig. 1). The number of bacteria in the collected samples was determined using a bacterial counter (Bacterial Counter, DUAA01NP-H, Panasonic Healthcare Co., Ltd., Tokyo, Japan).

The number of bacteria for each dog was defined as the sum of the measured values for the left and right maxillary fourth premolars. The mean \pm standard deviation of the number of oral bacteria collected at weeks 0 to 8 from 5 dogs in each group was compared using the Mann-Whitney $U$ test. A value of $P<0.05$ was considered statistically significant.

Figure 2 shows the number of oral bacteria in each week from weeks 0 to 8 for the 5 dogs in the S, SP, SB and SPB groups. The numbers of oral bacteria in all the groups were lower than the detection limit $\left(1 \times 10^{5}\right)$ at Week 0 . The number of oral bacteria in the $\mathrm{S}$ group exceeded $800 \times 10^{5}$ colony-forming units $(\mathrm{CFU}) / \mathrm{m} l$ at Week 1 , continued to increase until Week 3 and then decreased and became stable around $600 \times 10^{5} \mathrm{CFU} / \mathrm{ml}$. The number of oral bacteria in the $\mathrm{SP}$ group gradually increased and exceeded $600 \times 10^{5} \mathrm{CFU} /$ $\mathrm{m} l$ at Week 5, and then subsequently decreased. The number of oral bacteria was significantly lower in the SP group than the S group until Week 4, but the significant difference between the groups disappeared after Week 5. The number of oral bacteria in the dogs in the SB and SPB groups was significantly lower than in the $\mathrm{S}$ group throughout the study (Weeks 1 to 8). No significant difference in the number of oral bacteria was observed between the SPB and SB groups; however, a tendency toward lower numbers of bacteria was observed in the SPB group.

The most common method used to evaluate periodontal diseases of dogs is subjective assessment by a veterinarian; however, adequate quantitative criteria to diagnose such diseases in dogs have not been established. Koch's postulates are not applicable to periodontal diseases $[6,7]$; consequently, the bacteria cannot be specifically identified. However, periodontal diseases begin with bacterial colonization of the periodontal tissues, which leads to tissue destruction. Increase in the bacterial load in the periodontal tissues may increase the inflammatory response; eventually, the bacteria and resulting bacterial degradation products will destroy the tissues [7]. That is, the onset risk of periodontal diseases may increase when prevalence of oral organisms increases and vice versa. Therefore, this study used a bacterial counter with dielectrophoretic impedance measurement (DEPIM) to quantitatively evaluate the number of oral bacteria. This system captures bacteria in proximity to an electrode in liquids and aligns them along the electric field to form a bacterial chain, and a short circuit between the electrodes caused by the bacterial chain changes the impedance between the electrodes to determine bacterial density (Fig. 3). DEPIM is positively correlated with conventional culture methods, which enables dielectrophoresis of only viable organisms (i.e., excluding dead organisms), and provides the same overall analysis as the culture methods.

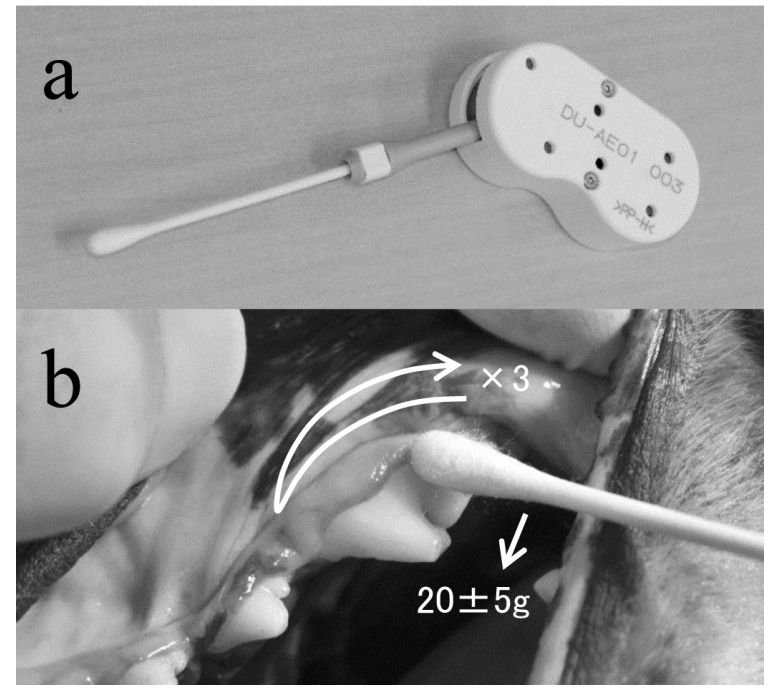

Fig. 1. Sampling from tooth surfaces using a sample collection device under constant pressure. A weight of $20 \pm 5 \mathrm{~g}$ was loaded on the buccal surface of the left and right maxillary fourth premolars using a sample collection device under constant pressure with a sterile cotton swab (a), and the buccal surface was rubbed back and forth 3 times using the cotton swab in a parallel position to the surface to collect samples (b).

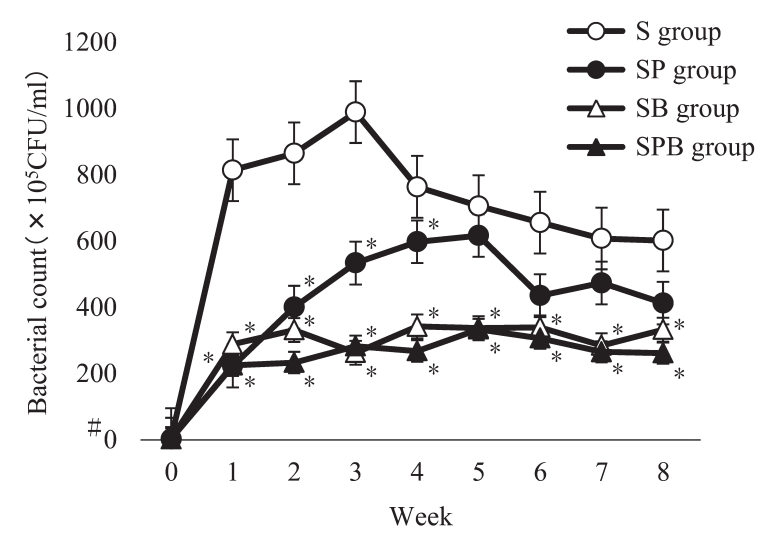

Fig. 2. Changes in the number of oral bacteria in each group. The number of oral bacteria in the S group increased until Week 3 , and subsequently decreased and became stable. On the other hand, the number of oral bacteria in the SP group gradually increased until Week 5 and subsequently decreased. The number of oral bacteria was significantly lower in the SP group than the S group until Week 4 , but the difference between the groups disappeared after Week 5 . The number of oral bacteria in the SB and SPB groups was significantly lower than that in the $\mathrm{S}$ group throughout the study period. *There was a significant difference compared with the $\mathrm{S}$ group at the same time $(P<0.05)$. " Lower than the detection limit $\left(1 \times 10^{5}\right)$.

In addition, DEPIM can be used to comprehensively detect and determine the number of bacteria $[3,4,8]$. This method is considered more suitable for determining the number of oral bacteria, which mainly consist of unidentified bacteria of various strains and difficult-to-culture bacteria $[1,10]$. In gen- 


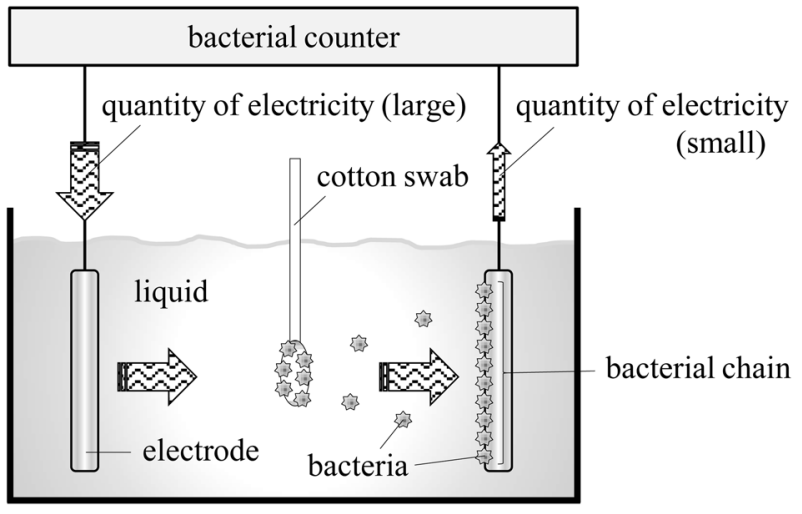

Fig. 3. Operating Principle of the Bacterial Counter. Bacteria in liquid align along the electric field to form a bacterial chain, and a short circuit between the electrodes caused by the bacterial chain changes the impedance between the electrodes to determine bacterial density.

eral, the samples for the bacterial counter are collected from the tongue in human experiments; however, in this study, the samples were collected from the buccal surface of the maxillary fourth premolars of the dogs, because dog tongues are likely to be exposed to the external environment [7].

In this study, the number of oral bacteria in the $\mathrm{S}$ group was lower than the detection limit at Week 0, exceeded 800 $\times 10^{5} \mathrm{CFU} / \mathrm{m} l$ at Week 1 , continued to increase until Week 3 , and subsequently decreased and became stable around $600 \times$ $10^{5} \mathrm{CFU} / \mathrm{ml}$. The increase at Week 1 after scaling suggested that scaling can remove oral bacteria (Week 0), but might also destroy the bacterial flora and result in an increase in the number of aerobic bacteria and facultative anaerobes. The subsequent decrease in the number of bacteria that stabilized around $600 \times 10^{5} \mathrm{CFU} / \mathrm{m} l$ may have occurred because of an increased number of anaerobic bacteria in the gingival sulci. The anaerobic oral bacterial flora may have been restored; thus, aerobic bacterial growth was inhibited [11], which also explained the decrease in the number of bacteria in the SP group after Week 5. Our findings suggested that the removal of plaque and calculi by scaling alone was not sufficient to prevent oral bacterial growth. The number of oral bacteria in the SP group gradually increased until Week 5 and exceeded $600 \times 10^{5} \mathrm{CFU} / \mathrm{ml}$, and then subsequently decreased. The number of bacteria was significantly lower in the SP group compared with the $\mathrm{S}$ group until Week 4, but no significant difference was observed after Week 5 . In fact, tooth surfaces were more likely to have bacteria after scaling, but then, they were smoothed by polishing. However, the effect was transient and lasted approximately 1 month. The number of oral bacteria in the SB and SPB groups was significantly lower than in the $\mathrm{S}$ group throughout the study (Weeks 1 to 8), showing that daily tooth brushing had an inhibitory effect on oral bacterial growth. Furthermore, no significant difference in the number of bacteria was observed between the SB and SPB groups, but the number of oral bacteria in the SPB group tended to be lower. This result suggested that a combination of brushing and polishing was more effective to smooth the tooth surfaces and prevent colonization compared with brushing alone, and polishing may enhance the effect of tooth brushing.

The results of this study that were obtained using a bacterial counter showed that the number of oral bacteria increased within 1 week after scaling alone, whereas after polishing, the number of oral bacteria was relatively unchanged after approximately 1 month. The number of oral bacteria was significantly lower for scaling combined with brushing compared with scaling alone, regardless of the presence or absence of polishing, suggesting that daily brushing can inhibit oral bacteria growth more effectively than scaling alone or scaling and polishing. Polishing may be effective only when it is combined with brushing. This study indicated that continuation of daily brushing was the best way to prevent the onset of periodontal diseases in dogs.

\section{REFERENCES}

1. Aas, J. A., Paster, B. J., Stokes, L. N., Olsen, I. and Dewhirst, F. E. 2005. Defining the normal bacterial flora of the oral cavity. $J$. Clin. Microbiol. 43: 5721-5732. [Medline] [CrossRef]

2. DuPont, G. A. 1998. Prevention of periodontal disease. Vet. Clin. North Am. Small Anim. Pract. 28: 1129-1145. [Medline] [CrossRef]

3. Enjoji, T., Uchida, S. and Tochukubo, F. 2011. Investigation of relationship among dielectric characteristic of microorganisms, active status of cell membrane and flow rate dependence using dielectrophoretic impedance measurement method. J. Inst. Electrostat. 35: 139-144.

4. Hamada, R., Suehiro, J., Nakano, M., Kikutani, T. and Konishi, K. 2011. Development of rapid oral bacteria detection apparatus based on dielectrophoretic impedance measurement method. IET Nanobiotechnol. 5: 25-31. [Medline] [CrossRef]

5. Hamp, S. E., Olsson, S. E., Farsø-Madsen, K., Viklands, P. and Fornell, J. 1984. A macroscopic and radiologic investigation of dental diseases of the dog. Vet. Radiol. 25: 86-92. [CrossRef]

6. Harvey, C. E. 1998. Periodontal disease in dogs: Etiopathogenesis, prevalence, and significance. Vet. Clin. North Am. Small Anim. Pract. 28: 1111-1128, vi. [Medline] [CrossRef]

7. Harvey, C. E. 2005. Management of periodontal disease: understanding the options. Vet. Clin. North Am. Small Anim. Pract. 35 : 819-836, vi. [Medline] [CrossRef]

8. Kikutani, T., Tamura, F., Takahashi, Y., Konishi, K. and Hamada, R. 2012. A novel rapid oral bacteria detection apparatus for effective oral care to prevent pneumonia. Gerodontology 29: e560-e565. [Medline] [CrossRef]

9. Mankodi, S., Berkowitz, H., Durbin, K. and Nelson, B. 1998. Evaluation of the effects of brushing on the removal of dental plaque. J. Clin. Dent. 9: 57-60. [Medline]

10. Marsh, P. D. 2004. Dental plaque as a microbial biofilm. Caries Res. 38: 204-211. [Medline] [CrossRef]

11. Uzel, N. G., Teles, F. R., Teles, R. P., Song, X. Q., Torresyap, G., Socransky, S. S. and Haffajee, A. D. 2011. Microbial shifts during dental biofilm re-development in the absence of oral hygiene in periodontal health and disease. J. Clin. Periodontol. 38: 612-620. [Medline] [CrossRef]

12. Van der Weijden, G. A., Timmerman, M. F., Danser, M. M. and Van der Velden, U. 1998. Relationship between the plaque removal efficacy of a manual toothbrush and brushing force. $J$. Clin. Periodontol. 25: 413-416. [Medline] [CrossRef] 